$\underset{4}{4}$

E N C R U C I J A D A

$35^{\circ}$ NÚMERO Mayo-Agosto 2020

REVISTA ELECTRÓNICA DEL

Revista Electrónica de

CENTRO DE ESTUDIOS EN

Centro de Estudios en Administración Pública de la

Facultad de Ciencias Políticas y Sociales,

ADMINISTRACIÓN PÚBLICA

Universidad Nacional Autónoma de México

DOI: http://dx.doi.org/10.22201/fcpys.20071949e.2020.35.71397

\title{
Gobierno abierto: buen gobierno o corrupción en México, 2013-2018
}

\author{
Miguel Ángel de Guadalupe Sánchez Ramos * \\ Shantay Castillo de la Rosa \\ Recibido: 10 de octubre de 2019 \\ Aceptado: 13 de enero de 2020
}

\section{Resumen:}

El objetivo del artículo es analizar la relación entre gobierno abierto, corrupción y disposición al buen gobierno en México, 2013 al 2018. Los intentos en México por construir un gobierno abierto son débiles y desiguales, asociados con más corrupción, lo que ha producido una disposición baja al buen gobierno. El gobierno mexicano ha desplegado acciones parciales y no como parte de una estrategia holística que condujera al mejoramiento general de las condiciones de vida. La parcialidad en los esfuerzos gubernamentales redunda en la menor acogida de efectividad por parte del ciudadano, produciendo desconfianza y mayor percepción de corrupción. Los niveles de descomposición son de tal nivel que el estado de derecho no se percibe como ligado a los elementos del gobierno.

\section{Palabras clave:}

Buen gobierno, efectividad, confianza, transparencia, participación colaborativa.

\begin{abstract}
:
The purpose is to analyze the relationship between open government, corruption and willingness to good governance in Mexico, 2013 to 2018. Attempts in Mexico to build an open government are weak and uneven, associated with more corruption, which has produced a low disposition to good government. The Mexican government has deployed partial actions and not as part of a holistic strategy that would lead to the general improvement of living conditions. The bias in government efforts results in the lower reception of effectiveness by the citizen, producing distrust and greater perception of corruption. The levels of decomposition are of such a level that the rule of law is not perceived as linked to the elements of government.
\end{abstract}

* Doctor en Ciencias Políticas por el Centro de Investigación y Docencia en Humanidades del Estado de Morelos. Miembro del Sistema Nacional de Investigación del Conacyt. Profesor de Tiempo Completo en la Universidad Autónoma del Estado de México, adscrito al campus Amecameca. Línea de investigación: Gobierno abierto. Correo electrónico: <masr35@hotmail.com>.

** Licenciada en Ciencias Políticas y Administración Pública por la Universidad Autónoma del Estado de México. Colaboradora en la Dirección Operativa de Transporte Público Individual de la Secretaría de Movilidad de la CDMX. 
Gobierno abierto: buen gobierno o corrupción en México, 2013-2018.

Miguel Ángel de Guadalupe Sánchez Ramos y Shantay Castillo de la Rosa

DOI: http://dx.doi.org/10.22201/fcpys.20071949e.2020.35.71397

\section{Keywords:}

Good governance, effectiveness, trust, transparency, collaborative participation

\section{Introducción}

El siglo XXI está demostrando que la función de gobierno cada vez se hace más complicada en coherencia a la complejidad social; por lo tanto, los sujetos que desarrollan la actividad de gobernar requieren mayores habilidades y capacidades para que el gobierno logre efectividad.

Un gobierno es efectivo cuando cumple dos características: 1) ser sensible, y 2) ser responsable (Putnam, 1994). Sensible es que sea capaz de entender o interpretar la legítima demanda de su comunidad. Responsable en dos sentidos: a) dar respuesta idónea a las necesidades, y b) responder, tener responsabilidad por sus decisiones y actos tomados. En esta tesitura, el ejercicio de gobernar puede tener dos caminos, mal gobierno o buen gobierno. El primero es aquel que está lejano de la representación, de la demanda sentida de la comunidad y es incapaz de dar respuesta, incluso hace lo posible por burlar la responsabilidad que le compete.

El buen gobierno, es la expresión sensata del arte de gobernar (Aguilar 2000), de hacerlo con decoro, con honestidad, con transparencia, con sensibilidad y dando la cara a su sociedad para responderle en sus demandas y responder por sus actos u omisiones (Bautista, 2017). Claro está que la división entre ambos caminos es muy débil, para el buen gobierno se requiere de personas con estabilidad y salud de todo tipo.

El régimen democrático anhela buen gobierno. Un gobierno democrático es complejo (Sánchez, 2017), supone capacidad de gobierno que promueve y no sólo acepta participación y deliberación constante, además ofrece trato igual y respetuoso a todos los ciudadanos reconociéndoles su derecho a manifestarse y escoger opciones así como proponer alternativas. Robert Dahl (1991) hace una explicación importante entre la participación y la deliberación en la formación de sociedades democráticas o poliárquicas como él las bautizó.

En la democracia representativa se necesita un modelo conversacional, dialógico, y que promueva sana interacción entre gobernantes y ciudadanos. Esta característica lo promete el gobierno abierto. En este sentido, el gobierno abierto es la estrategia para la construcción de buen gobierno por su esencia dialógica, interactiva. La intersubjetividad es la condición de la construcción social del conocimiento y de los asuntos públicos. 
Gobierno abierto: buen gobierno o corrupción en México, 2013-2018.

Miguel Ángel de Guadalupe Sánchez Ramos y Shantay Castillo de la Rosa

DOI: http://dx.doi.org/10.22201/fcpys.20071949e.2020.35.71397

En sentido inverso, un gobierno que está cerrado, que es opaco, que no promueve la participación y la colaboración sienta las condiciones para construir un Estado corrupto, que no sólo gobierno, deshonesto y con vicios en el ejercicio de la actividad gubernamental.

El estudiar, entonces, estas dos categorías en un gobierno, la de gobierno abierto y la corrupción, trasciende en el área académica y social, en la primera por hallar datos empíricos que pueden ofrecer el diagnóstico causal de la condición de buenos y malos gobiernos, ubicados en su contexto. La investigación es fundamental para el perfeccionamiento de los gobiernos y sus resultados, de no considerarlo así se está condenado a repetir los mismos errores.

En lo social, la segunda área de interés, es de suma importancia porque permite comprender la intersubjetividad que subyace en la construcción de los gobiernos abiertos o corruptos, y con esto tener elementos para proponer las mejoras respectivas a la dimensión de las características sociales. En la actualidad donde la sociedad del conocimiento está en constante ampliación y complejidad, el escrutinio de las acciones y decisiones gubernamentales es más público, más crítico, más exigente; de ahí que la sistematización de un diagnóstico puede ayudar a consolidar la observación, su comprensión y transformación.

El objetivo del presente es analizar la relación establecida entre gobierno abierto y corrupción en México, durante los años 2013 al 2018 y con esto la disposición al buen gobierno. Para dar cumplimiento a esto se cuenta con los siguientes apartados que ayudan en la exposición, en primer lugar se define el problema de la investigación; en un segundo momento se concisa la literatura que es fundamental para entender esta relación que se desea explorar; se expone la metodología que se ha seguido; se presentan los resultados obtenidos, para al final cerrar con la discusión y conclusiones que sostienen el nivel de la disposición al buen gobierno alcanzado en México frente a la relación entre el gobierno abierto y la corrupción.

\section{Planteamiento del problema}

México no se ha mostrado ajeno al crecimiento social y de complejidades que esto produce; lo singular del caso es la forma en cómo se ha atendido la relación entre gobernantes y gobernados, donde la estrategia de la apertura y la evolución de las tecnologías han ayudado para posicionar en la opinión pública los temas de corrupción, y de alguna manera han influido para ser perseguidos. La libertad de expresión ha adquirido naturalización en las redes sociales a través de las cuales se filtra la demanda de un gobierno bueno. 
Gobierno abierto: buen gobierno o corrupción en México, 2013-2018.

Miguel Ángel de Guadalupe Sánchez Ramos y Shantay Castillo de la Rosa

DOI: http://dx.doi.org/10.22201/fcpys.20071949e.2020.35.71397

Sin embargo, el asunto no es unilateral, requiere de un ejercicio de construcción social donde la idea de buen gobierno sea compartida, asimilada y ejercida por el Estado como el constructo de población y gobierno. La intersubjetividad es fundamental en esta construcción, porque las diversas manifestaciones públicas son su producto.

México ha iniciado desde los años 70 del siglo XX esquemas de liberalización y democratización que le han permitido avanzar en temas de alternancia en la representación y en el gobierno; empero no han sido suficientes para construir un escenario diferente. El autoritarismo no se ha ido del todo, sigue estando en la forma de dirigir de diversos espacios, no solo del gobierno o poder político, también se le encuentra en la cúpulas de las organizaciones, de los partidos políticos, escuelas, universidades y una serie de realidades sociales.

La corrupción es una práctica que no se ha podido desarraigar de la política en México y que, en obviedad, acarrea altos costos políticos, sociales, económicos y culturales. La Organización de los Estados Americanos puntualiza que la corrupción en México equivale al 10\% de su producto interno bruto; el Centro de Estudios Económicos del Sector Privado evalúa en 1.5 billones de pesos el costo de la corrupción mexicana (Casar, 2015). Entre los diferentes costos sobresale el tema de la desconfianza social que se ha construido hacía con la política y los políticos, subestimando la capacidad de la política en la formulación de grandes acuerdos y decisiones consensadas; grandes cantidades de dinero se ven referidas en los escándalos de corrupción y también constituyen sumas de magnitudes considerables al suponer los pequeños actos de corrupción, desde la cuota al agente vial en el crucero, hasta las propinas en la ventanillas o diezmos exigidos en las transacciones de adjudicación, que al ser muchos, ascienden a montos impactantes.

Los rankings sobre el tema, para el año 2017 ubican a México en el lugar 135 de 180; con base en el índice de Percepción de la Corrupción 2017 (Transparency International, 2017), la calificación de México empeoró un punto porcentual. En la región de América Latina y el Caribe está peor que Brasil, Argentina y Colombia, ocupando posiciones similares a las de Honduras y Paraguay (Transparency International, 2017). México está ubicado en el lugar 135 de 180 países, con un índice de 0.32 en ausencia de corrupción. Esto demuestra que estos últimos años ha habido un retroceso en materia de la percepción de la corrupción que empeora las condiciones de buen gobierno.

Si bien, la tendencia mundial de impulsar el gobierno abierto como estrategia de rendición de cuentas y de contención de la corrupción (OCDE, 2016), ha logrado propagación y firma de alianzas y compromisos gubernamentales, también es cierto que en México no se percata como una acción consolidada. El gobierno abierto se encuentra en el lugar 36 de 113 países, 
Gobierno abierto: buen gobierno o corrupción en México, 2013-2018.

Miguel Ángel de Guadalupe Sánchez Ramos y Shantay Castillo de la Rosa

DOI: http://dx.doi.org/10.22201/fcpys.20071949e.2020.35.71397

con un índice de 0.61 (WJP, 2018) en el ámbito federal, mientras que en las entidades federativas se aprecia diferencias en los avances. Los desniveles aún son más notorios en los gobiernos locales o municipales.

Como puede observarse, hay una relación negativa entre gobierno abierto y corrupción en México. El esfuerzo de la transparencia y acceso a la información no ha traído mejoras en la percepción del asunto de la corrupción, en suma el buen gobierno no permea a la población, y en consecuencia, hay niveles altos de insatisfacción.

La pregunta que ha guiado la investigación cuyo primer reporte se integra en este artículo es ¿cómo han sido los esfuerzos durante los años 2013-2018 por construir un gobierno abierto en México y qué relación guardan con la corrupción? La respuesta a esta cuestión y asumida como hipótesis estriba en que los intentos en México por construir un gobierno abierto son débiles y desiguales, en asociación con más corrupción entre los años 2013-2018, lo que ha producido una disposición baja al buen gobierno.

Las discusiones teóricas asientan sobre una relación positiva entre estas variables en estudio, donde la apertura del gobierno gira hacia la disminución de la corrupción y abona al buen gobierno (Bautista, 2016; Casar, 2015; Cahidez, 2016; Coronado, 2018; CLAD, 2016; Guadarrama, 2015). En ese sentido es oportuno recopilar lo más representativo de las disertaciones al respecto.

\section{Revisión de la literatura}

El gobierno abierto se sienta en el presupuesto de la apertura como un valor político connatural al espacio relacional, que utiliza la tecnología para lograr otros valores como la justicia, comunicación, eficiencia y colaboración (Jiménez, 2014) así como la participación, los cuales se logran con el reconocimiento de la importancia de la transparencia, acceso a la información pública y rendición de cuentas.

El Centro Latinoamericano para la Administración del Desarrollo (CLAD, 2016) expide una Carta que reconoce que el gobierno abierto ofrece una gestión pública eficaz, que garantiza la construcción de sociedades prósperas, equitativas y justas, lo que implica mayor interacción entre el Estado y la sociedad, basada en la confianza, transparencia, rendición de cuentas y participación cuya finalidad sea la justicia social y el bien común. Para eso es importante un Estado de Derecho que promueva el establecimiento de un buen gobierno. 
Gobierno abierto: buen gobierno o corrupción en México, 2013-2018.

Miguel Ángel de Guadalupe Sánchez Ramos y Shantay Castillo de la Rosa

DOI: http://dx.doi.org/10.22201/fcpys.20071949e.2020.35.71397

El gobierno abierto estriba en un conjunto de mecanismos y estrategias que contribuyen a la gobernanza pública y al buen gobierno, a través de principios como la transparencia, participación ciudadana, rendición de cuentas, colaboración, innovación y donde la ciudadanía está en el centro de las decisiones que cuente con políticas consensadas (CLAD, 2016; AGA, 2018).

La apertura conjugada con la transparencia, rendición de cuentas y participación colaborativa va a ser una estrategia para la disminución de la corrupción por la construcción de instituciones responsables, plurales e inclusivas.

Las tecnologías de la comunicación y de la información son el instrumento que facilita la construcción del gobierno abierto, sobre todo lo referente a la web 2.0, aquella que permite la comunicación y el diálogo. Con esta plataforma se garantiza que puede atenderse las demandas o consultas de manera fácil y a menor costo.

El tema de la corrupción en México ha sido estudiado por diferentes connotados académicos (Morris, 1992; Ramírez y Sánchez, 2013; Lomtiz, 2000; Martínez, 2002; Coronado, 2008; Pardinas, 2015; Casar, 2015; Martínez, 2015; Guadarrama, 2015) caracterizando el problema a diferentes causas, donde se destacan impunidad, los débiles marcos normativos y la percepción de que no son justas, la desigualdad social, inseguridad en el empleo, los malos salarios, endebles mecanismos de control en la administración pública, vicios o actitudes antiéticas, la propia codicia humana, y la falta de desarrollo moral, entre otras. Esto deja claro que se está frente a un problema complejo que corresponde más a escollos estructurales que sólo culturales.

La corrupción es el segundo problema que más preocupa a los mexicanos, $50.9 \%$ de población mexicana mayor de 18 años según el Instituto Nacional de Estadística y Geografía (2016), el cual considera que ésta existe al solicitar, aceptar, ofrecer o dar un bien o servicio, producto de una actuación ilegal derivada de un aprovechamiento indebido y para beneficio personal de un cargo, posición o responsabilidad pública o privada. Este fenómeno ocasiona una serie de desafecciones a la política, al gobierno y sus instituciones. La competitividad se ve detenida y afectada por la corrupción (Pardinas, 2015), así también se establece una baja calidad de las instituciones y del marco de derecho (Casar, 2015; Aguilar, 2000) como un fenómeno de círculo vicioso que cuenta con débiles normas, mismas que se favorecen y aumentan con la corrupción. Una buena ley requiere ser aplicada por un gobernante que tenga la fuerza extraordinaria para hacerla respetar (Aguilar, 2000).

La corrupción está presente en todas las regiones del país (Ramírez y Sánchez, 2013), la inseguridad jurídica, impunidad, discrecionalidad, patrimonialismo, falta de legitimidad, pérdida de confianza y la ineficiencia son constantes de la corrupción (Martínez, 2002). 
Gobierno abierto: buen gobierno o corrupción en México, 2013-2018.

Miguel Ángel de Guadalupe Sánchez Ramos y Shantay Castillo de la Rosa

DOI: http://dx.doi.org/10.22201/fcpys.20071949e.2020.35.71397

El combate a la corrupción presupone la existencia de buenos gobiernos, que desde lo moral, el estado de derecho y mejor justicia social promuevan valores públicos más sólidos y menos caminos de corrupción. El estudio del buen gobierno hace referencia a la calidad del gobierno, por lo que es importante ubicar con claridad el objeto de estudio, el ejercicio del gobierno y no su acceso a él; esta diferencia esencial coadyuva a centrar las características de la capacidad del gobierno para ser bueno o tener calidad gubernativa (Cejudo, Sánchez y Zabaleta, 2009). En estos términos el estudio encuentra similitud a las definiciones hechas por Diamond y Morlino (2005) al estudiar la calidad de la democracia.

El buen gobierno no se define a partir de un resultado, dado que un resultado no está condicionado por el ejercicio de la autoridad y tampoco un resultado es el reflejo de todo el ejercicio de poder y gobierno. Por lo tanto, la calidad del gobierno o el buen gobierno está dado por las características sostenidas en el ejercicio de la autoridad y no sólo en los resultados y mucho menos en la forma en cómo se accedió al poder (Cejudo, Sánchez y Zabaleta, 2009). El buen gobierno es la acumulación de valores (Bautista, 2007).

El buen gobierno no se debe a un momento específico de la historia, es un aprendizaje social e histórico y no sólo a posturas deductivas de la lógica (Villoria, 2011), y tiene que ver con la construcción social sobre los principios y valores de lo bueno y de la efectividad. No puede haber buen gobierno si la gente no cambia en sus hábitos y creencias (Villoria, 2011), por lo tanto, el bueno gobierno implica una sociedad informada, culta y dispuesta a hacer respetar sus valores. El mismo Manuel Villoria (2011) afirma que en las sociedades en donde hay desconfianza a la honestidad y se cree en la existencia de la corrupción del gobierno, entonces la falta de cumplimiento de la ley está expandida.

El buen gobierno implica atender las necesidades reales de la población, atender a los gobernados, la legitimidad que se gana con las urnas se sostiene con el ejercicio gubernamental (Giner y Sarasa, 1997). Un buen gobierno es democrático porque responde a las preferencias ciudadanas sin establecer diferencias políticas (Aguilar, 2000) ni promueve la división entre su sociedad, al contrario, busca la unidad y la no polarización social para controlar de mejor manera el conflicto que puede aminorar la sinergia de la comunidad para el desarrollo. Por lo tanto, desde el gobierno se impulsa un espíritu de formación que consiste en la construcción social del valor de la política, lo gubernamental y lo público.

El buen gobierno está caracterizado por la práctica de la eficiencia, eficacia, transparencia, rendición de cuentas, participación ciudadana, estado de derecho (Martínez, 2010). La compaginación de estos elementos es básica para el impulso del desarrollo económico y social. 
Gobierno abierto: buen gobierno o corrupción en México, 2013-2018.

Miguel Ángel de Guadalupe Sánchez Ramos y Shantay Castillo de la Rosa

DOI: http://dx.doi.org/10.22201/fcpys.20071949e.2020.35.71397

El buen gobierno tiene como pilares una buena administración y una buena burocracia; aquella se mueve en la eficacia, eficiencia, imparcialidad y honestidad; la burocracia se distingue por estar compuesta con personas competentes y profesionales.

\section{Metodología}

El estudio se constituye como longitudinal al analizar el comportamiento de gobierno abierto y corrupción en un lapso que va del 2013 al 2018, en donde su evolución es importante no sólo por tratarse de un gobierno sexenal en la presidencia de la República Mexicana, sino también por considerar los diversos reportes que miden estas categorías de análisis y definir la evolución o involución.

La información principalmente se recabó de bases de datos que han construido organizaciones públicas, tanto gubernamentales como de la sociedad civil, como por ejemplo el Instituto Nacional de Geografía y Estadística (INEGI), Centro de Estudios Sociales y Opinión Pública de la Cámara de Diputados (CESOP, 2018), Alianza para el Gobierno Abierto (AGA, 2018 y Open Government Pathership, 2018), Sistema Nacional Anticorrupción, Secretaría de la Función Pública, Instituto Mexicano para la Competitividad (IMCO, 2018), Transparencia Mexicana, Programa de Naciones Unidas para el Desarrollo (PNUD, 2018), International Transparency, entre otros.

A partir de la información recopilada en estas bases, se procedió a construir el dato que permitiera conocer el nivel de gobierno abierto y corrupción que se ha alcanzado en los diferentes ámbitos de gobierno en México. Con ese dato se procedió a la aplicación del análisis respectivo, según el método escogido.

Para poder demostrar la hipótesis se utilizó el método comparativo y estadístico. El primero se despliega con el establecimiento de parangones constantes entre las diversas acciones y mediciones que se hallan como resultado de evaluaciones que organismos públicos de la organización social e internacional realizan sobre el tema para los ámbitos de gobierno: federal, estatal y municipal. La comparación no solo responde al nivel de gobiernos versus gobiernos, sino también se compara la evolución que logra cada gobierno entre los años de estudio.

En el método estadístico se utilizó, como principales técnicas, las medidas de tendencia central y de dispersión, para conocer el grado de disparidad que existe en los esfuerzos por construir gobiernos abiertos y, en su caso, tolerar la corrupción; otra técnica fundamental para 
Gobierno abierto: buen gobierno o corrupción en México, 2013-2018.

Miguel Ángel de Guadalupe Sánchez Ramos y Shantay Castillo de la Rosa

DOI: http://dx.doi.org/10.22201/fcpys.20071949e.2020.35.71397

la comprobación de la hipótesis es la prueba de chi cuadrada que mide la existencia de asociación entre dos variables, así como la prueba de correlación entre variables.

Tres índices son centrales para demostrar lo expuesto, uno por cada dimensión:

a) sobre gobierno abierto

b) sobre corrupción

c) sobre la disposición al buen gobierno

Una vez que se construyó la base de datos y se procedió al análisis comparativo y estadístico se obtuvieron hallazgos que en el apartado siguiente se presentan. La exposición de estos se hace en tres esferas, primero se coloca la arena del gobierno abierto, posteriormente se exponen los resultados sobre corrupción, y finalmente, se presenta el resumen o balance sobre buen gobierno.

\section{Resultados}

a) Gobierno abierto

México ingresa a la Alianza para el Gobierno Abierto que convoca Estados Unidos con el liderazgo en la presidencia de este último país de Barack Obama, en septiembre de 2011, junto con siete países más: Brasil, Indonesia, Noruega, Filipinas, Sudáfrica, Reino Unido y Estados Unidos. Actualmente esa Alianza la integran 65 países.

El gobierno mexicano ha elaborado tres planes dentro de esta Alianza, el primero signado en el 2011 compromete el mejoramiento de los servicios públicos en 300 trámites y servicios federales, fortalecer el portal www.gob.mx, interoperabilidad de datos abiertos, e incrementar la rendición de cuentas (Presidencia de la República, 2011). El "Plan de Acción 2013-2015 México" reconoce que el gobierno abierto produce gobernanza, que es una forma de interacción novedosa con la comunidad donde se privilegian los emprendimientos y la generación de soluciones públicas. México se plantea cinco objetivos en lo que centra la promoción de transparencia y rendición de cuentas. Estas serían esenciales para combatir la corrupción. Los temas de los objetivos son: a) Gobierno centrado en la ciudadanía, en donde sus puntos de atención son la construcción de una página web (gob.mx), regular la transparencia, el registro de detenidos y desaparecidos, así como de beneficiarios; b) Presupuesto abierto y participa- 
Gobierno abierto: buen gobierno o corrupción en México, 2013-2018.

Miguel Ángel de Guadalupe Sánchez Ramos y Shantay Castillo de la Rosa

DOI: http://dx.doi.org/10.22201/fcpys.20071949e.2020.35.71397

tivo, en el que se centra prioridad en publicitar las compras abiertas, en red, el gasto transparente, seguimiento de obras; c) datos abiertos para el desarrollo, se propone una web para disponer de datos abiertos (datos.gob.mx), las materias privilegiadas son los carreteros, de la educación y la paz; d) empoderamiento y participación ciudadana, se impulsa la transparencia en lo respectivo a becas y apoyos que se producen con los programas gubernamentales; y e) gobernanza de recursos naturales, están los temas de desastres naturales, petróleo, minería y el medio ambiente (Presidencia de la República, 2014).

En el año 2015 se elabora el tercer plan de acción, para los años 2015-2018, en el que se reitera la disposición a mantener el gobierno abierto a través del establecimiento de los siguientes objetivos tematizados en: a) Derechos humanos y fortalecimiento del Estado de Derecho; b) Sistema Nacional Anticorrupción; c) Pobreza y desigualdad; d) Igualdad de género; e) Gobernanza de recursos naturales y cambio climático; f) Servicios públicos de salud; y g) Servicios públicos de agua (Presidencia de la República, s/f).

En el año 2013, el Barómetro de Datos Abiertos consideraba a México con valor de 40.3 puntos en el lugar 25. El primer lugar estaba ocupado por el Reino Unido con 100 puntos, seguido de Estados Unidos con 93.38, Suecia con 85.75, Nueva Zelanda con 74.34 y Noruega con 71.86 (World Justice Proyec, 2013).

Para el año 2016, en otra medición del Barómetro de Datos Abiertos, el Reino Unido contó con los 100 puntos y estuvo en el ranking 1, seguido de Canadá con 89.54 puntos, Francia con 85.13, y Estados Unidos con 81.62. México se encuentra con 73.45, lugar 11 (World Justice Proyec, 2018).

Lo que se desprende de estas mediciones internacionales es que México avanzó en este periodo de estudio 14 lugares, mejorando con sus acciones la condición de establecer o estar dispuesto a construir un gobierno abierto. Situación que se confirma cuando se revisa el avance anual logrado, según el mismo Barómetro, dado que en el 2014 logró 50.09 puntos y ubicarse en el lugar 24, para el año 2015 logra 61.76 puntos y ubicación en el lugar 16. Estos datos evidencian que los esfuerzos han logrado mejor posicionamiento en gobierno abierto al mexicano.

Al analizar esta dimensión de estudio en el contexto de las entidades federativas mexicanas se encuentra que el Centro de Investigación y Docencia Económicas A.C., en coordinación con Transparencia Mexicana publicaron en el 2017 "métrica de gobierno abierto", con promedios de dos pilares: transparencia y participación ciudadana. El índice de gobierno abierto hallado en este estudio para la federación es de 0.46 , por arriba del promedio nacional que es de 0.39 , 
Gobierno abierto: buen gobierno o corrupción en México, 2013-2018.

Miguel Ángel de Guadalupe Sánchez Ramos y Shantay Castillo de la Rosa

DOI: http://dx.doi.org/10.22201/fcpys.20071949e.2020.35.71397

con una desviación estándar de 0.05. La entidad mejor calificada en gobierno abierto es el de la Ciudad de México con un índice de 0.51, único que está por encima del resultado de la federación. Le sigue a la Ciudad de México, Guanajuato con 0.48, Jalisco con 0.45, Estado de México con 0.44 , Zacatecas y Aguascalientes con 0.43 . Mientras que los más bajos en este índice son Puebla y Tamaulipas con 0.34 , Oaxaca y Nayarit con 0.33 , Tlaxcala con 0.29 y Querétaro con 0.27 .

Al desglosar el índice de gobierno abierto a través de sus elementos, se encuentra que la participación ciudadana ha avanzado menos que la transparencia. En esta última la federación logra un índice de 0.59 , por arriba del promedio nacional que se encuentra en 0.46 , este índice como resultado de la calificación que el mismo gobierno se otorga; y un 0.64 como resultado de la apreciación ciudadana, por arriba del promedio nacional ciudadano que es de 0.54.

Según el gobierno, los estados mejor calificados en transparencia son: Ciudad de México (0.56), Jalisco y Sinaloa (0.52), Estado de México y Quintana Roo (0.51). Los estados con más bajo índice son: Baja California Sur (0.42), Durango (0.41), Tlaxcala (0.36), Hidalgo y Puebla (0.35), y Querétaro (0.32).

Según la ciudadanía, los mejores estados en transparencia son: Guanajuato (0.70), Ciudad de México (0.68), Estado de México (0.66), Morelos (0.64) Yucatán y Zacatecas (0.63). Los estados más bajos son: Baja California Sur y Puebla (0.47), Tamaulipas (0.43), Tlaxcala y Oaxaca (0.41), y Querétaro (0.32). Como se aprecia, los ciudadanos fueron más benévolos en su apreciación con respecto a los esfuerzos de calificación de la transparencia gubernamental.

En el tema de participación ciudadana medida desde el gobierno, el promedio nacional es de 0.23, la federación es de 0.31, los mejores estados son: Ciudad de México (0.47), Tabasco y Guanajuato (0.37), Veracruz y Coahuila (0.33), Sinaloa (0.31). Los más bajos son: Campeche, Quintana Roo y Colima (0.14), Chiapas (0.12), Tlaxcala (0.11), Guerrero (0.10) y Querétaro (0.09).

La participación ciudadana desde la apreciación ciudadana tiene los siguientes resultados, el promedio nacional es de 0.32, la federación 0.30; los mejores estados son: Aguascalientes (0.48), Sonora y Jalisco (0.47), Zacatecas (0.43), Michoacán, Quintana Roo, Coahuila y Estado de México (0.38); mientras que los estados con más bajo índice son: San Luis Potosí y Sinaloa (0.26), Hidalgo (0.25), Tabasco (0.24) Nayarit y Tamaulipas (0.22) y Morelos (0.21).

De este índice se desprende que el gobierno de la Ciudad de México es el mejor posicionado en el tema del gobierno abierto, le sigue Guanajuato y Jalisco, considerando el índice global y 
Gobierno abierto: buen gobierno o corrupción en México, 2013-2018.

Miguel Ángel de Guadalupe Sánchez Ramos y Shantay Castillo de la Rosa DOI: http://dx.doi.org/10.22201/fcpys.20071949e.2020.35.71397

el de cada elemento. Mientras que en la misma lógica, el gobierno que sobresale por ser mal calificado en gobierno abierto es Querétaro, seguido por Tlaxcala y Puebla.

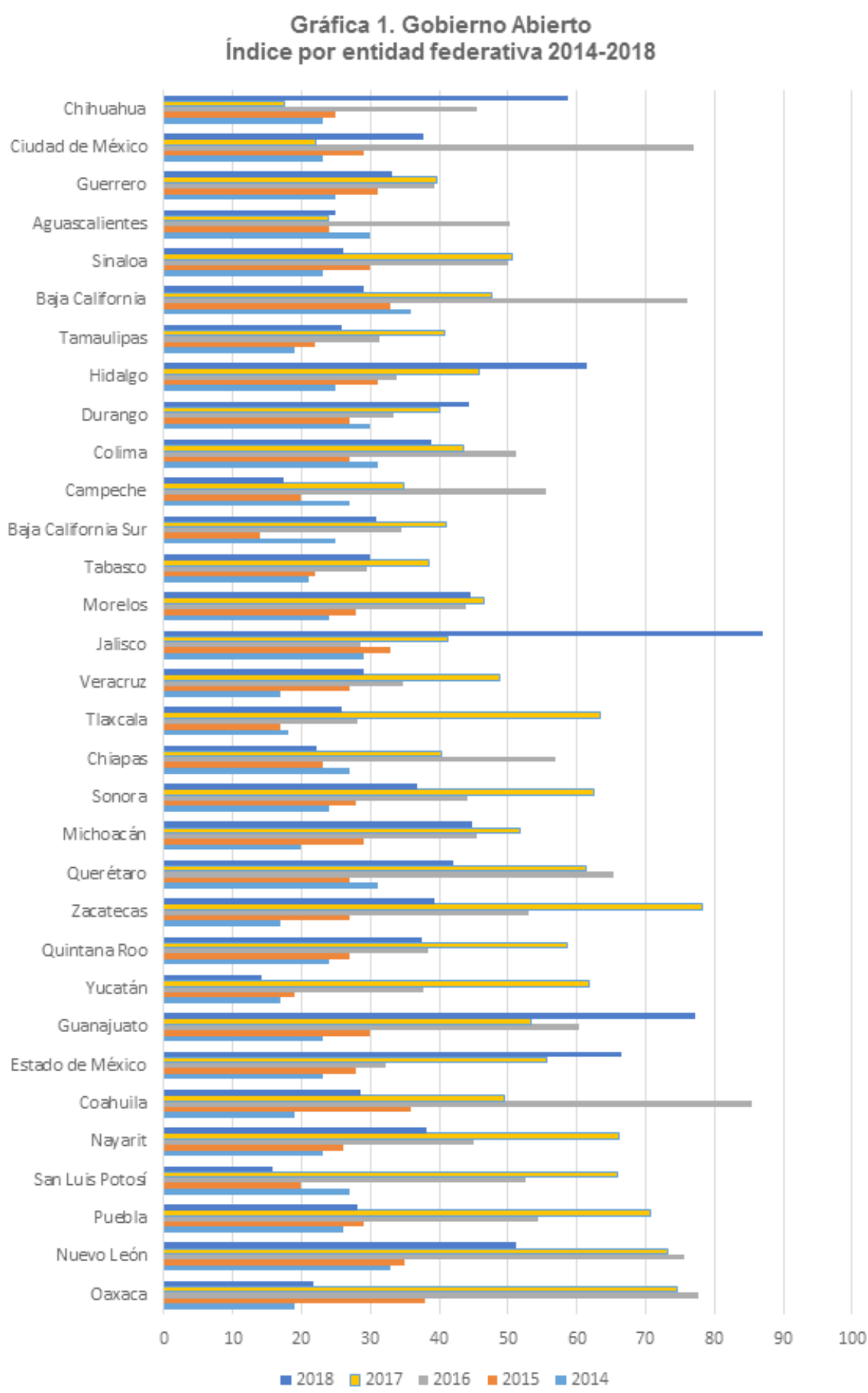

Fuente: Elaboración propia con base en datos de http://rodrigosandoval.mx/gobierno-abierto/ 
Gobierno abierto: buen gobierno o corrupción en México, 2013-2018.

Miguel Ángel de Guadalupe Sánchez Ramos y Shantay Castillo de la Rosa

DOI: http://dx.doi.org/10.22201/fcpys.20071949e.2020.35.71397

Una medición longitudinal realizada por Rodrigo Sandoval (2018) y publicada en la web, demuestra que los esfuerzos en las entidades federativas son diversos año con año, no se mantiene una línea firme, sólida y continua en gobierno abierto. En la gráfica 1 se observa las diversas variaciones que se han registrado en gobierno abierto a través de las mediciones a sus portales, tal como lo define en su metodología Sandoval (2018). La gráfica permite constatar el resultado de cuatro años, en los cuales el liderazgo no lo sostiene ninguna entidad, hay cambios. En el último año de la evaluación el mejor estado es Jalisco y le sigue Guanajuato, el primero ya muy completo en su apertura. Esta situación de altas diferencias evidencia que no se ha institucionalizado el gobierno abierto en el orden estatal y que está dependiendo de proyectos del titular del ejecutivo.

En una medición de Sandoval (2013) el mejor Estado es Nuevo León, seguido de Oaxaca, Baja California, Coahuila y Querétaro. Mientras que los de menor impacto está Tlaxcala, Baja California Sur, Chihuahua, Tabasco y Tamaulipas.

Entre las dos mediciones que se han presentado en este artículo existen diferencias, estas muy probable se deben a las metodologías singulares que se consideran en cada investigación; pero independientemente de esto, para objeto de la hipótesis planteada al inicio del estudio, se confirma que los esfuerzos sobre gobierno abierto son diversos, no responden a una política que esté homologada y que sea realmente una política de estado. Si las acciones fueran resultado de decisiones firmes y derivadas de un plan de acción claro e institucional, las mediciones no estarían tan diferentes.

En cuanto al gobierno abierto en los municipios es en donde se encuentra más dispersión en los avances que se tienen en la materia, no hay un Estado de la República que tenga homologados los proyectos y sus municipios estén en el mismo nivel de apertura. Un ejercicio de medición lo realiza la Academia Interamericana de Derechos Humanos (AIDH), organismo de la Universidad Autónoma del Estado de Coahuila, la cual hace una revisión en una muestra compuesta por 204 municipios, donde el máximo de apertura es 100 y el mínimo es 0, teniendo en cuenta los tres pilares: transparencia, participación y colaboración.

En esta medición realizada por AIDH se tiene que el municipio que logra un gobierno más abierto es Tlajomulco de Zúñiga, del Estado de Jalisco, el que logra 91 puntos, le sigue Tlaquepaque también de Jalisco con 86 puntos, Aguascalientes, Aguascalientes con 79 puntos, Guadalajara, Jalisco con 77 puntos, Torreón, Coahuila con 76 puntos. Pero el gran contraste es que hay municipios que tienen 0 puntos como es el caso de Fresnillo, Zacatecas; Hidalgo del Parral, Chihuahua; Tantoyuca, Veracruz; y Apizaco, Tlaxcala. 
El contraste, en los avances municipales hace que el promedio general de esa muestra se ubique en 24 puntos, con una desviación estándar de 15.6, lo que produce un coeficiente de variación de $63.14 \%$. Esto evidencia lo que se propuso como hipótesis, la gran diferencia que se establece entre los logros sobre gobierno abierto.

Sánchez y Martínez (2016) en estudio centrado en dos estados: México y Oaxaca demuestran que el gobierno abierto municipal está asociado con el índice de desarrollo humano, de tal suerte que en municipios con alto desarrollo cuentan con mejor disposición al gobierno abierto, en contraste, los municipios con menos desarrollo humano tienen nula o baja disposición al gobierno abierto.

\section{b) Corrupción}

De conformidad con datos de la Organización para la Transparencia Internacional que edita de forma anual, la percepción de la corrupción, según su índice, se mide en una escala de 0 a 100 donde el 0 es una percepción de altos niveles de corrupción y el 100 corresponde a niveles muy bajos de corrupción. En esta tesitura, las mediciones realizadas a México ubican a este país durante los años 2012-2017 en un promedio de 32.83 puntos con una desviación estándar de 2.6 puntos, lo que establece una variación del $5 \%$ y que de conformidad a la gráfica 2 se aprecia un aumento en la percepción de la corrupción.

\section{Gráfica 2. Ranking e índice de corrupción para México}

\section{2-2017}

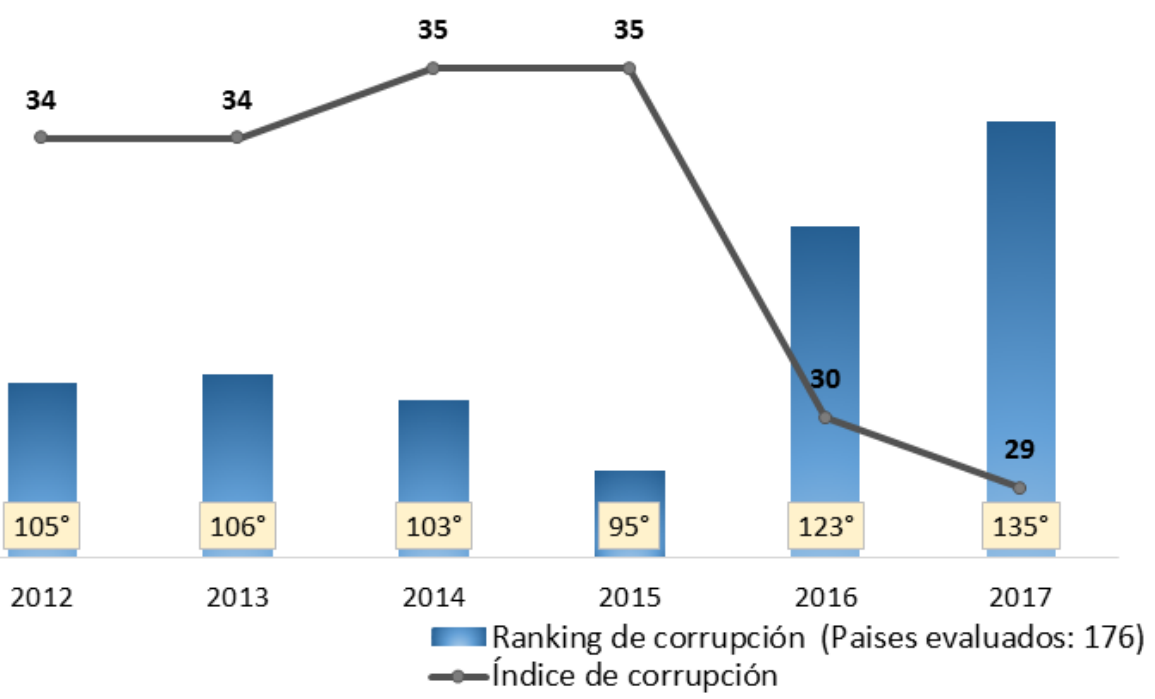

Fuente: Elaboración propia con información de Transparency International (2017) 
Esta misma Organización para la Transparencia Internacional complementa su índice con el establecimiento de rankings, mismos que se observan en la gráfica 2 para el caso mexicano, donde se desprende que el país ha pasado de un lugar 105 en el año 2012 a la posición 123 en el año 2016, y a la 135 en el año 2017, confirmando con esto que se trata de un país con altos índices de corrupción dado que el ranking califica a 176 países (Transparency International, 2017).

El Instituto Nacional de Geografía y Estadística (INEGI) estima la percepción ciudadana de corrupción, medida en frecuencia, de 0 a 100, donde 0 es menos frecuencia de corrupción y 100 más frecuencia de corrupción. En el año 2015, este Instituto aplicó una encuesta nacional sobre corrupción y los resultados se pueden observar en la gráfica 3, mismos que permiten apreciar que el promedio nacional es de $35 \%$. Los seis estados con mayor percepción de corrupción son Nuevo León, Sinaloa, Baja California, Baja California Sur, Durango y Ciudad de México; mientras que los seis menos corruptos: Campeche, Aguascalientes, Yucatán, Veracruz, Querétaro e Hidalgo. La diferencia entre el más corrupto y el menos es de 23.2 puntos.

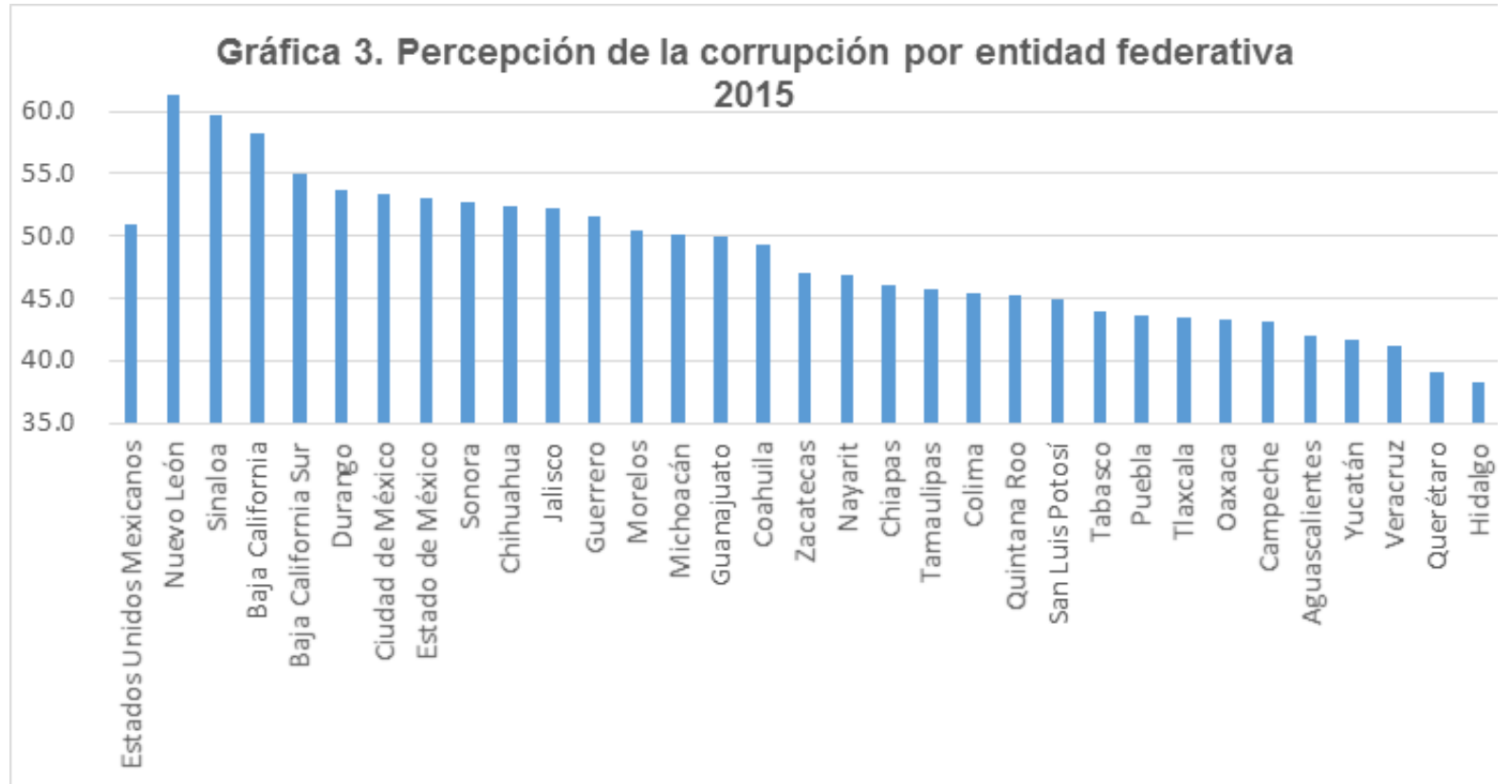

Fuente: Elaboración propia con información de INEGI (2016)

Al aplicar una prueba de hipótesis estadística de asociación entre el nivel de corrupción de una entidad federativa y el partido político que la gobierna, se obtuvo que a $95 \%$ de probabilidad 
Gobierno abierto: buen gobierno o corrupción en México, 2013-2018.

Miguel Ángel de Guadalupe Sánchez Ramos y Shantay Castillo de la Rosa

DOI: http://dx.doi.org/10.22201/fcpys.20071949e.2020.35.71397

no existe asociación entre estas dos variables, por lo que se puede afirmar que la corrupción no está asociada a un solo partido, sino que se presenta indistintamente en las diversas manifestaciones políticas.

Tabla 1. Tasa de prevalencia de corrupción por regiones, según trámites realizados en dependencias de gobierno federal, estatal y municipal, 2015.

(Víctimas por cada 100 mil habitantes)

\begin{tabular}{lcccc}
\hline Regiones & $\begin{array}{c}\text { Tasa de prevalen- } \\
\text { cia de corrupción } \\
\text { en trámites a nivel } \\
\text { nacional }\end{array}$ & $\begin{array}{c}\text { Tasa de prevalencia } \\
\text { de corrupción en } \\
\text { trámites del gobierno } \\
\text { federal }\end{array}$ & $\begin{array}{c}\text { Tasa de prevalencia de } \\
\text { corrupción en trámites } \\
\text { del gobierno estatal }\end{array}$ & $\begin{array}{c}\text { Tasa de prevalencia } \\
\text { de corrupción en } \\
\text { trámites del gobierno } \\
\text { municipal }\end{array}$ \\
\hline Estados Unidos Mexicanos & 12590 & 2729 & 17260 & 12645 \\
Región CentrWo & 13921 & 3706 & 19307 & 11936 \\
Región Noreste & 9534 & 1860 & 13140 & 10810 \\
Región Noroeste & 15747 & 2254 & 21137 & 18419 \\
Región Occidente & 11633 & 1936 & 15626 & 12463 \\
Región Sureste & 10173 & 2469 & 14055 & 9627 \\
\hline
\end{tabular}

Fuente: INEGI. Encuesta Nacional de Calidad e Impacto Gubernamental, 2015.

La tasa de prevalencia (víctimas de corrupción por cada 100 mil habitantes) medida por el INEGI está concentrada en la tabla 1, de la cual se aprecia que en los trámites federales la región más corrupta es el centro, debido en gran medida a que la mayoría de las oficinas del gobierno federal se ubican en esta región. La prevalencia más alta en las autoridades estatales se ubica en la región noroeste, seguida por la región centro. Ahora bien, la prevalencia más alta en las autoridades municipales se localiza en la región noroeste, igual que en la estatal, seguida por la región occidente, estas dos regiones son las que se conocen como las concentradoras de los carteles de la droga.

La prevalencia varía según la autoridad que se mida y también la región en donde se referencie. La autoridad con menos tasa de prevalencia es la federal con promedio de 2445 , con desviación estándar de 746 y un coeficiente de variación de 0.3052; seguida por la autoridad municipal con promedio de 12651, una desviación estándar de 3403 y un coeficiente de variación de 0.2690; y la más alta tasa la registra la autoridad estatal con un promedio de 16653, desviación estándar de 3438, y un coeficiente de variación de 0.2064 . Frente a esto se aprecia que la autoridad estatal tiene la más alta prevalencia e inclusive con menor variación, es decir, las entidades federativas están calificadas de forma muy similar, mientras que la autoridad federal cuenta con el coeficiente de variación más alto, por lo que se puede inferir que hay diferencia en sus trámites y, por lo tanto, en sus actos de corrupción. No todas las dependencias federales actúan de igual forma. 
Gobierno abierto: buen gobierno o corrupción en México, 2013-2018.

Miguel Ángel de Guadalupe Sánchez Ramos y Shantay Castillo de la Rosa

DOI: http://dx.doi.org/10.22201/fcpys.20071949e.2020.35.71397

En cuanto a las regiones, la que tiene la menor tasa de prevalencia es la del noreste con promedio de 8603 , desviación estándar de 5955, coeficiente de variación de 0.6921; le sigue la región sureste con un promedio de 8717 , desviación estándar de 5846, coeficiente de variación de 0.6706; seguida por la región occidente con un promedio de 10008, desviación estándar de 7167, y coeficiente de variación de 0.716; la penúltima región es la centro con promedio de 11650, desviación estándar de 7804, coeficiente de variación de 0.6699; la región más alta en prevalencia es la noroeste con promedio de 13937, desviación estándar de 10208, coeficiente de variación de 07324. Para el caso, la región más alta en tasa de prevalencia también es la que cuenta con la mayor dispersión relativa.

Ahora bien, en la tasa de incidencia (actos de corrupción por cada 100 mil habitantes), según el INEGI (2016) en el año 2015 se registra para México 30097 puntos, el estado con mayor incidencia es el Estado de México con 62160 puntos en su tasa, seguido por Sinaloa con 58232, Chihuahua con 36472, Sonora con 33256, y Baja California Sur con 31604 puntos. Mientras que los estados con menor tasa de incidencia son: Colima con 9230 puntos, seguido por Nayarit con 9879, Tamaulipas con 10441 puntos, Veracruz con 10461 puntos, y Zacatecas con 13252 puntos.

EI INEGI (2016) reporta que en su encuesta del 2015 se registró que $80 \%$ de los ciudadanos percibieron que en las instituciones públicas se dan con mayor frecuencia actos de corrupción, donde la policía y los partidos políticos son los identificados como más corruptos. En esta encuesta con análisis por entidad federativa, en 24 estados los policías son mencionados como más corruptos, en 6 son los partidos políticos, en 1 los gobiernos municipales y 1 los gobiernos estatales. Situación similar se recupera de la encuesta del mismo orden y hecha por el mismo INEGI en 2013, en donde 27 estados mencionan en primer lugar a los policías, y 5 estados son los partidos políticos.

En la comparación entre las dos encuestas, la del año 2013 y las del año 2015, los cinco estados que aumentaron en corrupción son: Baja California, Durango, Colima, Baja California Sur y la Ciudad de México, mientras que los cinco estados que disminuyeron en corrupción son: Tabasco, Veracruz, Puebla, Tamaulipas y Estado de México. Estos datos pueden ser contradictorios frente a los escándalos periodísticos que señalaron a algunos gobernantes de los últimos estados como actores de actos de corrupción de gran calado, como es el caso del ex gobernador de Veracruz, sin embargo son los datos que se encuentran consignados a través de la encuesta de INEGI.

La asociación existente entre partido político y grados de corrupción en los estados que gobiernan es débil, esto en atención a una prueba de chi cuadrada cuyo valor es de 18.206, una 
significancia asintótica bilateral de 0.052, lo que permite afirmar que al $90 \%$ de confianza hay asociación entre las dos variables mencionadas: partido político y corrupción, pero una vez que se busca el grado de intensidad de esa asociación a través de una prueba de Tau-c de Kendall es de -0.218 , lo que define que es débil esa asociación, o válida sólo para el $21 \%$ de los casos. Por lo tanto, no se puede sostener que la corrupción esté asociada a un partido o a dos en específico; es más amplia y complicada la manifestación de la corrupción.

Utilizando la prueba de correlación estadística, a través de la $\mathrm{R}$ de Pearson, se obtiene que hay una correlación positiva y significativa entre los grados de corrupción del año 2013 y los del año 2015, con una R igual a 0.746, lo que equivale a decir que es fuerte la correlación.

De igual forma hay correlaciones positivas entre los grados de corrupción del 2015 con el índice de educación medido en el índice de desarrollo humano (IDH) 2012, con una R igual a 0.347, crece el grado de educación y creció la corrupción.

El ingreso, medido en el IDH, y el grado de corrupción 2015, también guardan correlación con una $\mathrm{R}$ igual a 0.451, más ingreso más corrupción.

En general el IDH está correlacionado positivamente con el grado de corrupción 2015, con una r igual a 0.365 , más desarrollo humano y más corrupción.

El índice de marginación y el grado de corrupción están correlacionados con una R de Pearson igual a -0.397, menos marginación a más corrupción. Con estos datos se nota una paradoja, el desarrollo alienta la corrupción.

c) Balance del Buen gobierno

Las mediciones hechas sobre esta variable de estudio son realizadas por diversas organizaciones, destacando los resultados publicados por el Banco Mundial por haber integrado un análisis multivariable a partir de las respectivas mediciones de efectividad en el gobierno, estabilidad política y ausencia de violencia o terrorismo, calidad regulatoria, estado de derecho, responsabilidad y voz, y control de la corrupción. 
Gobierno abierto: buen gobierno o corrupción en México, 2013-2018.

Miguel Ángel de Guadalupe Sánchez Ramos y Shantay Castillo de la Rosa

DOI: http://dx.doi.org/10.22201/fcpys.20071949e.2020.35.71397

Gráfica 4. Indicadores de buen gobierno en México

(2012-2016)

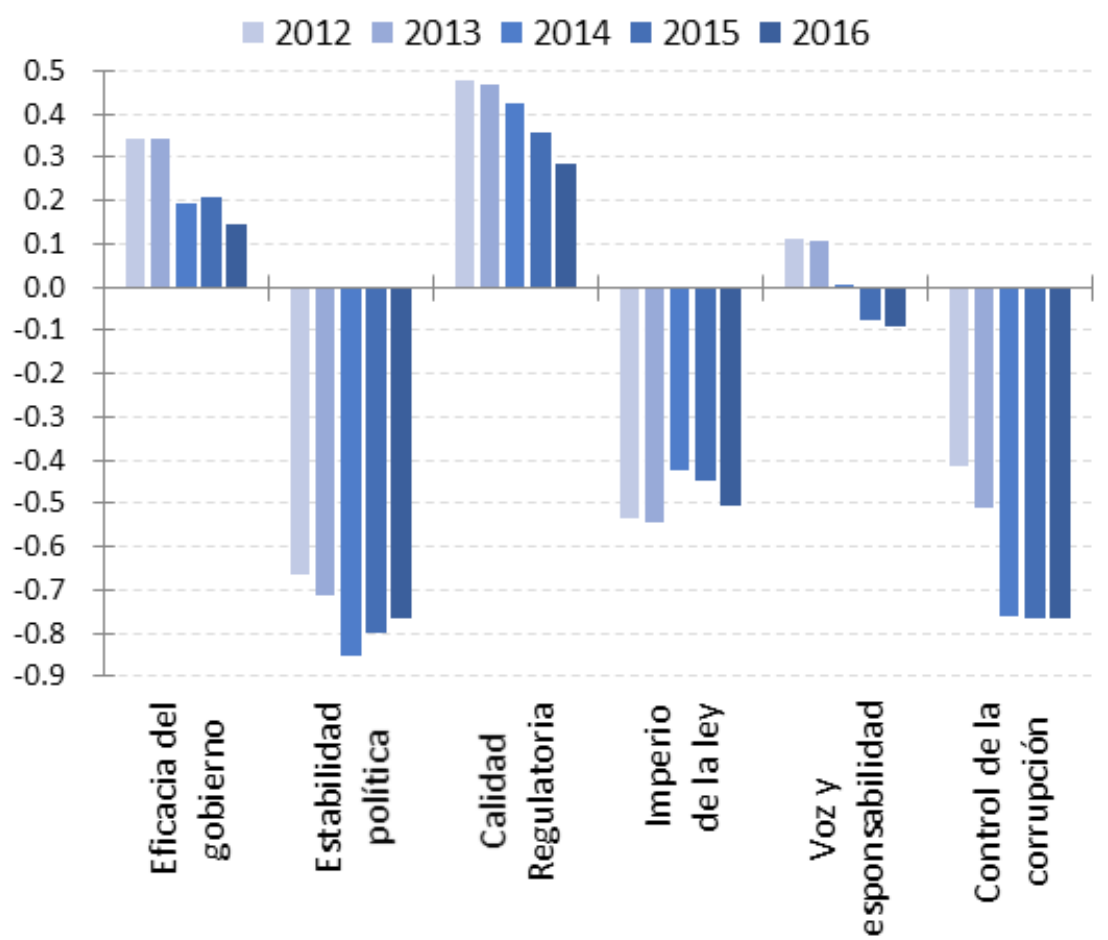

Nota: Puntaje en unidades de una distribución normal estándar, es decir, que va de aproximadamente -2.5 a 2.5.

Fuente: Elaboración propia con estimaciones del Banco Mundial

En cuanto a la efectividad del gobierno, en atención a la gráfica 4 se nota que en su estimación:

a) Corresponde a una puntuación ubicada en una escala como media, al oscilar entre 0.3 y $0.1 ; y$

b) Hay una tendencia hacia la baja, al ir de 0.3 en el año 2012 a una puntuación de 0.1 en el año 2016;

En la arena de la estabilidad política y ausencia de violencia y terrorismo, se aprecia:

a) Las puntuaciones son negativas y se ubican dentro de la escala de nivel medio, oscila entre el -0.7 y el -0.9 ;

b) Hay una tendencia a la baja al pasar de -0.7 a -0.8 .

La calidad regulatoria, como otra dimensión del buen gobierno, tiene el siguiente comportamiento: 
a) Las puntuaciones se ubican en la zona del nivel medio, del lado positivo, al corresponder entre 0.5 y 0.3 ;

b) Hay una tendencia a la baja, al pasar de 0.5 en el año 2012 a 0.3 en el año 2016.

El rol del derecho o estado de derecho, según la gráfica 4, tiene el siguiente comportamiento:

a) Las puntuaciones se ubican en la zona del nivel medio, pero del lado negativo, al fluctuar entre -0.4 y -0.5 ;

b) Hay una sensible estabilidad en su comportamiento al registrar en el año 2012 una puntuación de -0.5, en los años 2014 y 2015 regresar a -0.4 y en el año 2016 de nuevo registrar -0.5 .

En cuanto a la rendición de cuentas se puede observar lo siguiente:

Se ubica también en la zona del nivel medio, fluctuando entre el 0.1 y el -0.1;

a) Hay una tendencia a la baja al ir de 0.1 en el año 2012 a -0.1 en el año 2016.

En la materia de control de la corrupción se observa:

a) De igual manera que las anteriores dimensiones, del lado negativo, las puntuaciones se encuentran en la zona del nivel medio, al fluctuar entre -0.4 y -0.8 .

b) Hay una tendencia muy clara a la baja al pasar de -0.4 en al año 2012 a -0.8 en el año 2016, aunque según la gráfica se aprecia un estancamiento en este último valor a partir del año 2014. 
Gobierno abierto: buen gobierno o corrupción en México, 2013-2018.

Miguel Ángel de Guadalupe Sánchez Ramos y Shantay Castillo de la Rosa

DOI: http://dx.doi.org/10.22201/fcpys.20071949e.2020.35.71397

Tabla 2. Matriz de componentes rotados ${ }^{a}$

\begin{tabular}{|l|c|c|}
\hline \multirow{2}{*}{} & \multicolumn{2}{|c|}{ Componente } \\
\cline { 2 - 3 } & 1 & 2 \\
\hline Calidad regulativa & .983 & .163 \\
Efectividad del gobierno & .983 & .163 \\
Responsabilidad & .880 & .342 \\
\cline { 2 - 3 } $\begin{array}{l}\text { Control de corrupción } \\
\text { Estado de derecho }\end{array}$ & .778 & .611 \\
\cline { 2 - 3 } $\begin{array}{l}\text { Estabilidad política y ausencia de } \\
\text { violencia }\end{array}$ & -.072 & -.982 \\
\cline { 2 - 3 } & .493 & .800 \\
\hline
\end{tabular}

Método de extracción: Análisis de componentes principales.

Método de rotación: Normalización Varimax con Kaiser.

a. La rotación ha convergido en 3 iteraciones.

Fuente: Elaboración propia a partir de datos del Banco Mundial

En la intención por explicar el buen gobierno como categoría de análisis y al partir de la información ofrecida por el Banco Mundial, se procede a realizar una reducción de variables, por el método de análisis de componentes, de tal suerte que el análisis factorial que se obtiene permite identificar dos componentes, mismos que se observan en la tabla 2. A partir de esta tabla es evidente que el buen gobierno mexicano, o la disposición al buen gobierno mexicano se explica a través de dos componentes principales:

1) En el primer componente hay cuatro variables que lo explican por su importante relación, las cuales son calidad regulativa $(0.983)$, efectividad gubernamental o del gobierno (0.983), responsabilidad $(0.880)$, y control de corrupción (0.778)

2) En el segundo componente hay dos variables que por su importancia explican, las cuales son: estabilidad política y ausencia de violencia (0.8), y control de la corrupción (0.611). 
Gobierno abierto: buen gobierno o corrupción en México, 2013-2018.

Miguel Ángel de Guadalupe Sánchez Ramos y Shantay Castillo de la Rosa DOI: http://dx.doi.org/10.22201/fcpys.20071949e.2020.35.71397

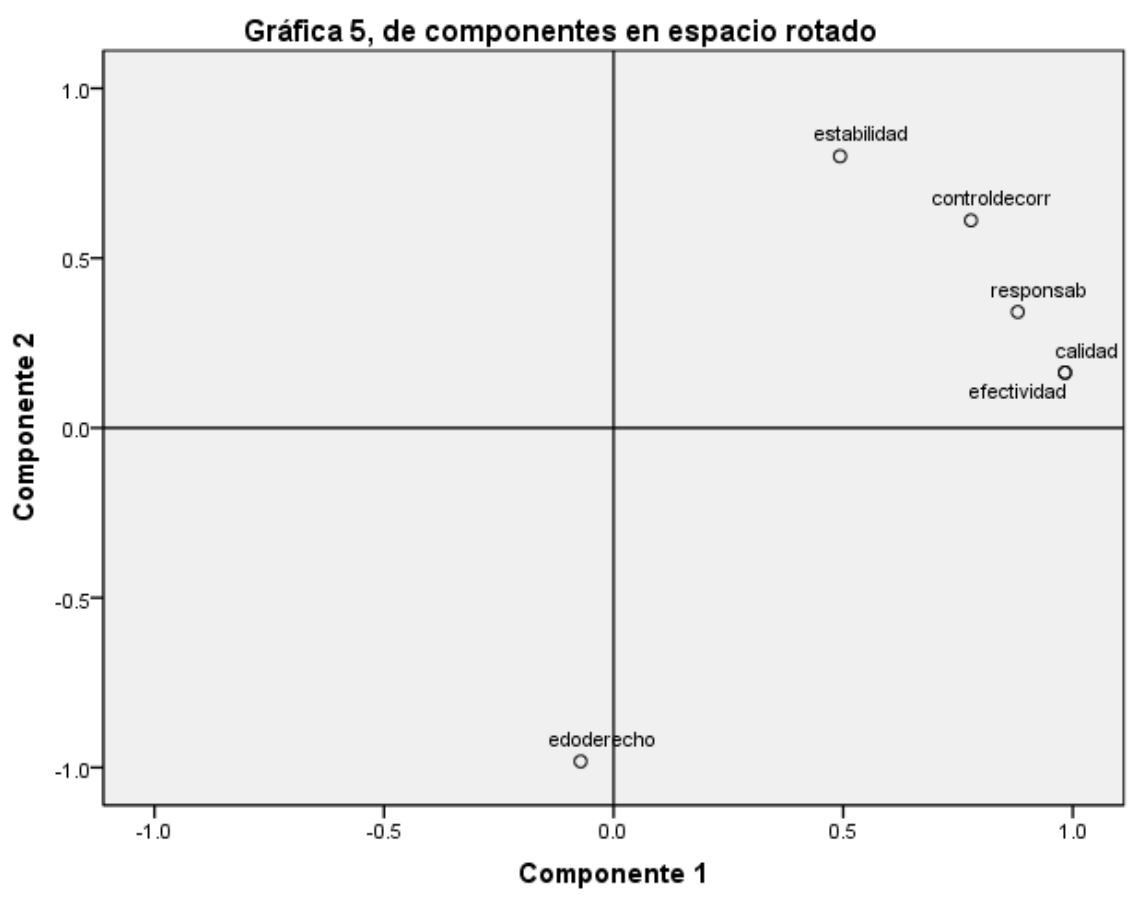

Fuente: Elaboración propia a partir de la base de datos del Banco Mundial

Por lo tanto, el primer componente se nombra como el propio del buen gobierno, por ver directamente con la efectividad del mismo; mientras que el segundo componente se nombra de estabilidad política, propiamente dicha al involucrarse la paz.

En la gráfica 5, que corresponde a la saturación del análisis factorial, se observa que el componente 2, nombrado para este efecto de estabilidad política, está explicado por la estabilidad política y ausencia de violencia, y el control de la corrupción.

El primer componente, llamado para este trabajo como el de efectividad gubernamental, está fuertemente sostenido por la relación que se da principalmente entre la efectividad, la calidad regulativa y la responsabilidad.

El estado de derecho es una variable que para el caso está aislada, producto de la desconfianza ciudadana y de que en las acciones de gobierno no se aprecia al Derecho como el instrumento de la certeza. 
Gobierno abierto: buen gobierno o corrupción en México, 2013-2018.

Miguel Ángel de Guadalupe Sánchez Ramos y Shantay Castillo de la Rosa

DOI: http://dx.doi.org/10.22201/fcpys.20071949e.2020.35.71397

\section{Discusión}

Los resultados demuestran que México ha asumido compromiso por ser un gobierno abierto, y ha desplegado acciones en ese sentido; sin embargo no hay una institucionalización de un plan sólido que impregne en los tres ámbitos de gobierno. Lo que se nota son esfuerzos desiguales en toda la manifestación del gobierno mismo. Prevalece el sentido de gobierno abierto igual a más transparencia, olvidando que también se trata de una participación colaborativa, la gran ausente en los esfuerzos del gobierno abierto mexicano. Así también lo confirma el estudio realizado por el Centro de Investigación y Docencia Económica (CIDE) y el Instituto Nacional de Transparencia, Acceso a la Información y Protección de Datos Persona (INAI) que coordinó Guillermo Cejudo (2017), el cual enfatiza que han habido avances en la transparencia en términos que responden a más esfuerzos legales, pero muy poco se ha avanzado en participación ciudadana. De manera similar lo asienta Chaidez (2016) que concluye que la participación ciudadana ha sido la gran ausente en los esfuerzos del gobierno mexicano al no promover las decisiones públicas con participación y colaboración.

El esfuerzo del gobierno federal mexicano es evidente a través de su participación en la Alianza para el Gobierno Abierto, pero limitada a mayor transparencia y presencia en la web. Sin embargo, las gestiones no responden a una estrategia definida de gobierno abierto direccionado a contener la corrupción y a construir un buen gobierno, son más resultado de tendencia que de convicción política. El gobierno mexicano considera que el gobierno abierto debería resolver problemas públicos mejorando la calidad de vida y generando beneficios sociales (OCDE, 2016), empero su estrategia no está encaminada a hacer realidad este concepto, sus trabajos están limitados y el esfuerzo no es general en todos los ámbitos de la administración pública.

Las acciones de los gobiernos estatales y municipales son dispares, correspondiendo más a los estilos personales del gobernante que a un plan o programa que institucionalice y norme el gobierno abierto. Los hallazgos sobre la disparidad de esfuerzos también son evidentes en Cejudo (2017), Chaidez (2016). Valenzuela y Bojórquez (2016) destacan que hay más de un modelo de implementación del gobierno abierto, lo que ha sido evidente en los resultados expuestos arriba, que no existe una política institucional que impulse las acciones de gobierno abierto en México. Por lo tanto, las diversas mediciones realizadas en la materia acreditan que no hay logros sostenidos.

El caso de los municipios registra la mayor dispersión, hay una gran variabilidad de esfuerzos, incluso al interior de los propios estados de la República. Situación que confirma la hipótesis establecida, la cual estriba en que hay una diferencia en los logros de gobierno abierto. Los 
Gobierno abierto: buen gobierno o corrupción en México, 2013-2018.

Miguel Ángel de Guadalupe Sánchez Ramos y Shantay Castillo de la Rosa

DOI: http://dx.doi.org/10.22201/fcpys.20071949e.2020.35.71397

municipios mejor ubicados en temas como el desarrollo humano tienen mejor gobierno abierto (Sánchez y Martínez, 2016), este hallazgo en México contrasta con lo localizado en Chile donde las variables socioeconómicas no están asociadas a los logros municipales de gobierno abierto (Hernández, 2016).

Se aprecia que las innovaciones logradas en un periodo no persisten, no hay continuidad en los esfuerzos desplegados. Esto es debido a la ausencia de una estrategia de políticas sobre gobierno abierto. No es exclusivo del gobierno local en México, ya Pau y Piana (2017) sostienen situación similar de innovaciones no perseverantes en Buenos Aires. Esta situación hace más complicado el trabajo de consolidar un gobierno abierto municipal.

La percepción de la corrupción ha crecido entre el mexicano, 8 de cada 10 considera que las instituciones públicas realizan con mayor frecuencia actos de corrupción. Los estados que han aumentado en esta percepción de más corrupción son Baja California, Durango, Colima, Baja California Sur y la Ciudad de México, mientras que los cinco estados que disminuyeron su percepción son Tabasco, Veracruz, Puebla, Tamaulipas y Estado de México. De manera aparente el aumento se ubica del centro al norte y la disminución del centro al sur. Estos datos van en contra sensu de lo hallado por Sour (2017) que expresa que los esfuerzos de la administración pública por innovar han contribuido para mejorar la percepción de la corrupción. Sí hay innovación pero no es la suficientemente efectiva para recuperar la confianza ciudadana y cambiar su percepción con respecto a la corrupción. Esto en gran parte estriba en una deficiente estrategia de comunicación que despliegan los gobiernos por lo que los ciudadanos desconocen lo que sus gobiernos realizan y, sí conocen sobre escándalos de corrupción.

Las distribuciones territoriales de la corrupción no son fortuitas, están asociadas a la presencia fuerte de organizaciones delincuenciales y de narcotráfico en el centro al norte. A la vez existe la paradoja de que grandes escándalos cubiertos por la prensa tienen que ver con diversos gobernantes de diversas latitudes territoriales, como un reflejo más fiel de la creciente corrupción en la clase política mexicana.

Los resultados apuntan a que entre menor es la marginación de los lugares se halla más corrupción. A mayores niveles educativos más corrupción. Lo que llevaría a establecer el corolario que el desarrollo económico está emparejado, en México, con la corrupción. Esto es preocupante en el sentido de que puede entenderse que el desarrollo en México se logra a partir del torcimiento de la ley.

Las acciones en materia de buen gobierno son también dispersas, no homogéneas, por lo tanto no se consolida la percepción de un buen gobierno. La tendencia de la efectividad se 
aprecia a la baja, igual que la estabilidad política, la rendición de cuentas y la calidad regulatoria. El estado de Derecho no es creíble en México. Si no se produce una percepción de buen gobierno se amplía la desconfianza en él y por lo tanto sus acciones no cuentan con la legitimidad necesaria para producir buenos resultados y satisfacción generalizada.

Se confirma que cuando hay desconfianza en la honestidad y en las propias instituciones políticas, es natural que se crea que hay corrupción en el gobierno (Villoria, 2011), dado que los resultados evidencian la tendencia a la alta en este periodo de estudio de la percepción de la corrupción, además de ir en incremento las tasas de desconfianza a la autoridad y al gobierno en general.

La construcción social del valor político y honestidad se impulsa desde el seno del gobierno, de ahí que los diversos símbolos, signos utilizados son importantes para la generación de confianza, para establecer gobiernos creíbles, buenos gobiernos.

El hecho de que estén avanzando los esfuerzos del gobierno por ser abierto y que la percepción de la corrupción aumente, quiere alertar que las estrategias de uno no se emparejan a la misión de servicio y búsqueda de legitimidad por desempeño. Esta situación evidencia la falta de una estrategia de buen gobierno, cuyas acciones están encaminadas y centradas en la ciudadanía.

La alternativa para combatir la corrupción no estriba en la creación y reproducción de órganos o comités, sino en una estrategia transversal que esté presente en todas las acciones cotidianas del individuo, desde la creación de sus imaginarios así como en la tarea vivencial de su cotidianidad. El valor de la anticorrupción es una construcción social que se recarga en la interacción intersubjetiva de todos los miembros de la sociedad, por lo que requiere espacios de recreación, innovación y creatividad. Mayor participación y colaboración son necesarias, pero antes la internalización es urgente para poder ponderar el escollo y la magnitud del valor ético en entredicho. 
Gobierno abierto: buen gobierno o corrupción en México, 2013-2018.

Miguel Ángel de Guadalupe Sánchez Ramos y Shantay Castillo de la Rosa

DOI: http://dx.doi.org/10.22201/fcpys.20071949e.2020.35.71397

\section{Conclusiones}

El objetivo de este artículo se cumplió al relacionar al gobierno abierto con la corrupción en México durante 2013 al 2018, destacando que los esfuerzos por impulsar el gobierno abierto han aumentado, pero también ha aumentado la percepción de la corrupción, por lo que se tiene una relación negativa entre estas dos variables de análisis. Esta relación negativa produce la percepción de una disposición baja al buen gobierno.

Se confirma la hipótesis en el sentido original de su construcción, los intentos en México por construir un gobierno abierto son débiles y desiguales, en asociación con más corrupción entre los años 2013-2018, lo que ha producido una disposición baja al buen gobierno.

El gobierno mexicano del periodo de estudio, se concluye, desplegó acciones parciales y no como parte de una estrategia holística que condujera al mejoramiento general de las condiciones de vida. La parcialidad en los esfuerzos gubernamentales redunda en la menor acogida de efectividad por parte del ciudadano, produciendo desconfianza y mayor percepción de corrupción.

Los niveles de descomposición son de tal nivel que el estado de derecho no se percibe como ligado a los elementos del gobierno. La ley y el derecho no garantizan la certidumbre de la acción gubernamental, de ahí que el gobierno abierto bajo los principios de transparencia, participación y colaboración pueda ser una estrategia para la gobernanza que reconstruya la recuperación del estado de derecho como símbolo circunscribe la acción de gobernados y gobernantes.

El gobierno abierto centrado en el ciudadano, que cuenta con una estrategia y se conduce con honestidad y transparencia, ocasiona que la participación y colaboración se conviertan en realidad, provocando que la confianza ciudadana se recupere y de manera colectiva se construya un buen gobierno.

La conjugación del trinomio gobierno abierto, anticorrupción y buen gobierno es fundamental para la legitimación y la recuperación de la credibilidad social en la política, de forma tal que en colaboración se construyan políticas que redunden en el mejoramiento de las condiciones de vida. Las acciones gubernamentales exitosas son las que están dirigidas al perfeccionamiento de las formas de vida asegurando, que logran promover la participación y colaboración de los diversos sectores, situación que asegura la contención de conductas agresivas o corruptas.

El Estado de Derecho es una construcción social que es responsabilidad de todos. El gobierno debe cumplir y hacer cumplir, el pueblo debe asegurarse de que se aplique y contribuir para que eso sea realidad. Un país con muchas normas es imagen de que tiene muchas conductas 
Gobierno abierto: buen gobierno o corrupción en México, 2013-2018.

Miguel Ángel de Guadalupe Sánchez Ramos y Shantay Castillo de la Rosa

DOI: http://dx.doi.org/10.22201/fcpys.20071949e.2020.35.71397

dañinas para la sociedad, pero también es reflejo de que se trata de un conjunto social que no respeta la norma por eso necesita más reglas. El reto es enorme para que el Estado de Derecho sea una expresión reconocida y vivida.

\section{Fuentes de información}

Aguilar, F. (2000), Moral pública en los procesos de buen gobierno, México: Plaza y Valdez.

AGA, Alianza para el Gobierno Abierto, México. Disponible en: http://gobabiertomx.org/ (Fecha de consulta: 12 de mayo del 2018).

Bautista, O. (2007), "Ética y política: Valores para un buen gobierno", México: Universidad Nacional Autónoma de México, disponible en: http://ri.uaemex.mx/bitstream/ handle/20.500.11799/58623/art-\%c3\%a9ticaypolitica-TI.pdf?sequence=1\&isAllowed=y (Fecha de consulta: 10 de abril de 2018).

Casar, M.A. (2015), "La corrupción: enemigo público \# 1", en, La corrupción en México Transamos y no avanzamos, Instituto Mexicano para la Competitividad, México: Índice de competitividad internacional.

Cejudo, G., \& Sánchez, G., \& Zabaleta, D. (2009), "El (casi inexistente) debate conceptual sobre la calidad del gobierno", en Política y Gobierno, XVI (1).

Cejudo, G. (Coord.). (2017), Métrica de gobierno abierto. Resultados 2017. México: Centro de Investigación y Docencia Económicas/ Instituto Nacional de Transparencia, Acceso a la Información y Protección de Datos Personales.

Centro de Estudios Sociales y de Opinión Pública, disponible en web:www.diputados.gob-mx/ cesop (Fecha de consulta 23 de febrero de 2018).

CLAD, (7 y 8 de julio de 2016), Carta iberoamericana de gobierno abierto. Obtenido de la Carta iberoamericana de gobierno abierto, disponible en web:http:// www.funcionpublica.gov.co/eva/admon/files/empresas/ZW1wcmVzYV83Ng==/ archivos/1470417708_350c543cc190276e5a10618fd50a3245.pdf (Fecha de consulta: 23 de noviembre del 2018).

Coronado, G. (2008), "Discourses of Anti-corruption in Mexico: Culture of Corruption or Corruption of Culture?", en Journal of Multidisciplinary International Studies, DOI:https:// doi.org/10.5130/portal.v5i1.479.

Chaidez, M. (2016), "El gobierno abierto en México: ¿Un nuevo modelo de Gobernanza?,” en Encrucijada, Revista electrónica del Centro de Estudios en Administración Pública, (24), 
Gobierno abierto: buen gobierno o corrupción en México, 2013-2018.

Miguel Ángel de Guadalupe Sánchez Ramos y Shantay Castillo de la Rosa

DOI: http://dx.doi.org/10.22201/fcpys.20071949e.2020.35.71397

disponible en https://docs.google.com/viewerng/viewer?url=http://www.revistas.unam. mx/index.php/encrucijada/article/viewFile/57768/51202 (Fecha de consulta: 10 de abril de 2018).

Dahl, R A. (1991), La poliarquía participación y oposición, Madrid: Tecnos.

Diamond, L. Morlino, L. (2005), Assessing the quality of democracy, USA: The Johns Hopkins University Press.

Giner, S. y Sarasa, S. (1997), Buen gobierno y política social, España: Ariel.

Guadarrama, M. (2015), "Corrupción y descomposición de la representación política", en La corrupción en México Transamos y no avanzamos, Instituto Mexicano para la Competitividad, México: Índice de competitividad internacional.

Hernández, J. (2016), "Diseño Institucional para el Gobierno Abierto Municipal: Propuesta de Medición y Análisis del caso Chileno", en Documentos y Aportes en Administración Pública y Gestión Estatal, 16(27).

INEGI (9 de diciembre de 2016), Estadística a propósito del día internacional contra la corrupción, México: Instituto Nacional de Estadística y Geografía.

IMCO, Instituto Mexicano para la competitividad A.C. México, disponible en web:http://imco. org.mx/indices/competitividad_internacional_2015/resultados/introducción (Fecha de consulta: 27 de mayo del 2018).

Jiménez, C. (2014), "Smart Government": un paso más allá del Gobierno Abierto, Recuperado de XIX Congreso Internacional del CLAD sobre la Reforma del Estado y de la Administración Pública, Quito, Ecuador, 11- 14 nov. 2014.

Lomnitz, C. (2000), Vicios públicos, virtudes privadas: la corrupción en México, México: Centro de Investigaciones y Estudios Superiores en Antropología Social/ Porrúa.

Martínez R. (2002), "Las políticas de la OCDE en la reforma de la administración pública y la lucha contra la corrupción en México". Conferencia llevada a cabo en el VII Congreso Internacional del CLAD sobre la Reforma del Estado y de la Administración Pública, Lisboa, Portugal.

Martínez C. (2010), "Los indicadores de buen gobierno en México y en el trabajo legislativo", Obtenido de Centro de Estudios Sociales y de Opinión Pública, disponible en web: http:/ www3.diputados.gob.mx/camara/content/download/241383/682729 (Fecha de consulta: 12 de marzo del 2018).

Morris, S.D. (1992), Corrupción y política en el México contemporáneo, México: Siglo XXI editores. 
Gobierno abierto: buen gobierno o corrupción en México, 2013-2018.

Miguel Ángel de Guadalupe Sánchez Ramos y Shantay Castillo de la Rosa

DOI: http://dx.doi.org/10.22201/fcpys.20071949e.2020.35.71397

Martínez A. (2015), "Desigualdad y corrupción ¿Cómo romper el círculo vicioso?”, en La corrupción en México Transamos y no avanzamos, Instituto Mexicano para la Competitividad, México: Índice de competitividad internacional.

OCDE (2016), Panorama de las administraciones públicas: América Latina y el Caribe 2017, París: OCDE, http://dx.doi.org/9789264266391-es

Open Government parthership (2018), Guía de gobierno de abierto, USA.

Pardinas, J. E. (2015), "Impunidad, corrupción y competitividad", en, La corrupción en México Transamos y no avanzamos, Instituto Mexicano para la Competitividad, México: Índice de competitividad internacional.

Pau, M. y Piana, R.S. (2017), "Políticas de gobierno abierto en los deliberativos municipales" en Camaou, A. y Pagani M. L., Debates teóricos y metodológicos actuales sobre las políticas públicas, La Plata: Facultad de Humanidades y Ciencias de la Educación.

PNUD. Prácticas de gobierno abierto y transparencia para el cumplimiento de los ODS. PNUD, disponible en: http://www.mx.undp.org/content/mexico/es/home/operations/projects/ democratic_governance/cumplimiento-de-los-ods-en-mexico---gobierno-abierto.html (Fecha de consulta 28 de noviembre del 2018).

Presidencia de la Republica (2011), "Alianza para el Gobierno Abierto Plan de Acción de México", disponible en: http://aga.ifai.mx/SitePages/EstamosHaciendo.aspx (Fecha de consulta: 07 de septiembre del 2018).

Presidencia de la República (2014), "Plan de acción 2013-2015 de la Alianza para el gobierno abierto", disponible en: http://aga.ifai.mx/Noticias/default.aspx (Fecha de consulta: 07 de septiembre del 2018).

Presidencia de la República. (s/f), "Tercer Plan de acción nacional de México en la alianza para el gobierno abierto", disponible en: https://www.gob.mx/cms/uploads/docs/Plan-deAccion-2016-2018.pdf (Fecha de consulta: 07 de septiembre del 2018).

Putnam, R. (1994), Para hacer que la democracia funcione. La experiencia italiana en descentralización administrativa, Caracas, Venezuela: Galac.

Ramírez L., \& Sánchez I. (2013), "Crecimiento económico, corrupción e instituciones en México”, Nóesis. Revista de Ciencias Sociales y Humanidades, 22 (43). DOI:https://doi. org/10.20983/noesis.2013.1.4

Sánchez, M, \&. Martínez, H. (2016), "Gobierno Local Abierto: Diagnóstico en México y Oaxaca" en Revista Iberoamericana de las Ciencias Sociales y Humanísticas, 5(10). DOI:https:// doi.org/10.23913/ricsh.v5i10.90 
Sánchez, M. (2017), "Gobierno abierto, una estrategia innovadora para el governance local”, en Olvera García, J y Contreras Orozco, L., Administración pública y sociedad. Una necesaria relación de proximidad. México: Porrúa/ Universidad Autónoma del Estado de México.

Sandoval, R. (2013), Ranking de Transparencia 2013. Ranking de gobierno abierto, disponible en: http://rodrigosandoval.mx/gobierno-abierto/ (Fecha de consulta: 15 de noviembre del 2018)

Sandoval, R. (2018), Ranking de Transparencia 2018. Ranking de gobierno abierto, disponible en: https://u-gob.com/ranking-de-portales-estatales-de-transparencia-2018/ (Fecha de consulta: 15 de noviembre de 2018)

Sour, L. (2017), "Gobierno electrónico y corrupción en México (2005-2010)”, en Innovar, 27(66), 123-136. Doi: 10.15446/innovar.v27n66.66807.

Transparency international (2017), Índice de Percepción de corrupción, disponible en: http:// www.transparency.org/news/feature/corruption_perceptions_index_2016 (Fecha de consulta. 10 de octubre del 2018).

Villoria, M. (2011), “¿Más libertad o más felicidad? El buen gobierno del siglo XXI, en Revista del CLAD Reforma y Democracia, (51).

Valenzuela, R. \&, Bojórquez, J. (2016), "Modelos de implementación del gobierno abierto en México" en Luna, I. (Ed,).Gobierno Abierto el valor social de la información pública, México: Universidad Nacional Autónoma de México.

World Justice Proyec (2013), “The Open Data Barometer", disponible en http://opendatabarometer.org/?_year=2013\&indicator=ODB (Fecha de consulta: 05 de enero del 2019).

World Justice Proyec (2018), "The Open Data Barometer", disponible en: http://opendatabarometer.org/?_year=2016\&indicator=ODB\&lang=es (Fecha de consulta: 05 de enero del 2019). 
Gobierno abierto: buen gobierno o corrupción en México, 2013-2018.

Miguel Ángel de Guadalupe Sánchez Ramos

DOI: http://dx.doi.org/10.22201/fcpys.20071949e.2020.35.71397

\section{$\sum_{-1}$}

\section{E N C R U C I J A D A \\ REVISTA ELECTRÓNICA DEL \\ CENTRO DE ESTUDIOS EN \\ ADMINISTRACIÓN PÚBLICA}

$35^{\circ}$ NÚMERO MAYO-ABRIL 2020

Revista Electrónica del

Centro de Estudios en Administración Pública de la

Facultad de Ciencias Políticas y Sociales,

Universidad Nacional Autónoma de México

UNIVERSIDAD NACIONAL AUTÓNOMA DE MÉXICO

Rector: Dr. Enrique Luis Graue Wiechers

Secretario General: Dr. Leonardo Lomelí Vanegas

Secretario Administrativo: Ing. Leopoldo Silva Gutiérrez

Abogada General: Dra. Mónica González Contró

\section{FACULTAD DE CIENCIAS POLÍTICAS Y SOCIALES}

Directora Interina: Dra. Carola García Calderón

Secretario General: Mtro. Arturo Chávez López

Secretario Administrativo: Lic. Juan Manuel López Ramírez

Jefe de la División de Estudios de Posgrado:

Dra. Laura Hernández Arteaga.

Jefa de la División de Educación Continua y Vinculación:

Mtra. Alma Iglesias González

Jefa de la División del Sistema Universidad Abierta y Educación a

Distancia: Lic. Yazmín Gómez Montiel

Jefa de la División de Estudios Profesionales:

Mtra. Rosa Merlín Rodríguez

Coordinador del Centro de Estudios en Administración Pública:

Dr. Adán Arenas Becerril

Coordinador de Informática:

Ing. Alberto Axcaná de la Mora Pliego

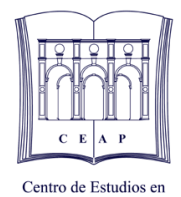

Centro de Estudios en FCPYS UNAM

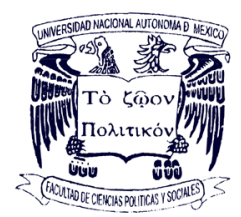

LA REVISTA

Director de la Revista:

Dr. Maximiliano García Guzmán

Secretario Técnico de la Revista:

Mtro. César C. Dionicio

Consejo Editorial:

Dr. Alejandro Navarro Arredondo

Dr. Arturo Hernández Magallón

Dr. Carlos Juan Núñez Rodríguez

Dra. Fiorella Mancini

$\operatorname{Dr}(\mathrm{c})$. Eduardo Villarreal

Dr. Roberto Moreno Espinosa

Diseño, integración y publicación electrónica: Coordinación de Informática, Centro de Investigación e Información Digital, FCPyS-UNAM. Coordinación de producción: Alberto A. De la Mora Pliego. Diseño e Integración de la publicación: Rodolfo Gerardo Ortiz Morales. Programación y plataforma Web: Guillermo Rosales García.

ENCRUCIJADA REVISTA ELECTRÓNICA DEL CENTRO DE ESTUDIOS EN ADMINISTRACIÓN PÚBLICA, Año 11, No.35, mayo-agosto 2020 , es una publicación cuatrimestral editada por la Universidad Nacional Autónoma de México a través de la Facultad de Ciencias Políticas y Sociales y el Centro de Estudios en Administración Pública, Circuito Mario de la Cueva s/n, Ciudad Universitaria, Col. Copilco, Del. Coyoacán, C.P. 04510, México, D.F., Tel. (55) 56229470 Ext. 84410 , http://ciid.politicas.unam.mx/encrucijadaCEAP/, ceap@politicas.unam.mx. Editor responsable: Dr. Maximiliano García Guzmán. Reserva de Derechos al uso Exclusivo No. 04-2011011413340100-203, ISSN: 2007-1949. Responsable de la última actualización de este número, Centro de Estudios en Administración Pública de la Facultad de Ciencias Políticas y Sociales, Mtro. César C. Dionicio, Circuito Mario de la Cueva s/n, Ciudad Universitaria, Col. Copilco, Del. Coyoacán, C.P. 04510, México D.F., fecha de la última modificación, 04 mayo de 2020.

Las opiniones expresadas por los autores no necesariamente reflejan la postura del editor de la publicación. Se autoriza la reproducción total o parcial de los textos aquí publicados siempre y cuando se cite la fuente completa y la dirección electrónica de la publicación. 